\title{
Controle Baseado em Quatérnio para Estabilização Simultânea da Orientação e Posição de Veículos Omnidirecionais *
}

\author{
Victor M. S. Peixoto* Rafael S. Castro* \\ Guilherme A. Pimentel* Aurelio T. Salton** \\ * PUCRS - Grupo de Automação e Controle de Sistemas, \\ Av. Ipiranga 6681, Porto Alegre, RS, Brazil, 90619-900 \\ (e-mail: victor.peixoto@edu.pucrs.br, rafael.castro@pucrs.br, \\ guilherme.pimentel@pucrs.br) \\ ** UFRGS - Departamento de Engenharia Elétrica, \\ Av. Osvaldo Aranha 103, Porto Alegre, RS, Brazil, 90035-190 \\ (e-mail: aurelio.salton@ufrgs.br)
}

\begin{abstract}
This paper presents a systematic approach able to design state-feedback controllers for simultaneous attitude and position stabilization of mecanum-wheeled omnidirectional planar vehicles. The system rotational motion is described by quaternions, leading to a differentialalgebraic dynamic model. From this representation, the control design is performed by a convex optimization problem subject to linear matrix inequalitiy constraints. These conditions guarantee the asymptotic stability and exponential convergence of the system trajectories with respect to the desired origin coordinate. A numeric example illustrates the functionality of the proposed approach.

Resumo: Este artigo apresenta um método sistemático para síntese de controladores de realimentação de estados para veículos omnidirecionais planares com rodas do tipo mecanum, visando a estabilização simultânea da orientação e posição do veículo. O movimento rotacional do sistema é descrito por quatérnios, o que permite a representação dinâmica do veículo na forma algébrico-diferencial. A partir dessa representação, o projeto do controlador é realizado através de um problema de otimização convexo sujeito a restrições na forma de desigualdades matriciais lineares. Estas condições garantem a estabilidade assintótica e a convergência exponencial das trajetórias do sistema em relação à coordenada de origem desejada. Um exemplo numérico é apresentado para demonstrar a funcionalidade do método de controle proposto.
\end{abstract}

Keywords: Omni-wheel Vehicle, Quaternions, Differential Algebraic Representation, Linear Matrix Inequalities.

Palavras-chaves: Veículo com Rodas Omnidirecionais, Quatérnios, Representação Algébrico-Diferencial, Desigualdades Matriciais Lineares.

\section{INTRODUÇÃO}

Apresentada por Trofino (2000), a representação algébricodiferencial (do inglês Differential Algebraic Representation, ou DAR) propõe-se a representar de forma geral sistemas que possuem funções racionais para os estados e incertezas paramétricas. Em seu artigo, o autor utiliza DAR em conjunto com condições baseadas em desigualdades matriciais lineares (do inglês Linear Matrix Inequalities) numa análise que garante estabilidade quadrática do sistema. Em Coutinho et al. (2004), novas condições são adicionadas, propondo um projeto de controle de custo garantido para o sistema. Um método para análise de estabilidade entrada-saída e um controle via realimentação de saída para sistemas não lineares sujeitos a distúrbios são

\footnotetext{
* O presente trabalho foi realizado com apoio da Coordenação de Aperfeiçoamento de Pessoal de Nível Superior - Brasil (CAPES) Código de Financiamento 001
}

apresentados em Coutinho et al. (2008). Uma análise de estabilidade regional robusta é vista em Coutinho et al. (2009), garantindo a estabilidade exponencial local dada uma região politópica. Oliveira et al. (2012) introduzem em suas análises saturação na entrada do sistema, propondo um controle via realimentação de estados utilizando LMIs. Em Castro et al. (2017) é introduzido um método sistemático de análise de estabilidade para sistemas não lineares racionais com controle de regulação de saída utilizando DAR e LMIs.

É possível observar que o estado da arte mostra a busca de generalizações de análise e controle para uma classe de sistemas não lineares. Salton et al. (2017) utiliza destes conceitos para aplicação mais específica, propondo uma solução ao problema de análise e controle de corpos rígidos rotativos, comum em pesquisas de sistemas não lineares, iniciando estudos para sistemas específicos. Neste mesmo artigo, utiliza-se quatérnios para representar o modelo do 
sistema, permitindo a representação em DAR e subsequente síntese de controle utilizando LMIs.

O presente trabalho propõe a aplicação de um método de controle de realimentação de estados para veículos omnidirecionais com rodas mecanum, considerando as nãolinearidades presentes na dinâmica do sistema, isto é, sem utilizar qualquer procedimento de linearização. $O$ fato deste tipo de veículo permitir inúmeras manobras, como deslocamento lateral sem rotacionar e rotação em torno do próprio eixo sem se deslocar, o torna ideal para diversas aplicações indoor, como para empilhadeiras ou cadeiras de roda (Naing et al., 2019). Pesquisas recentes utilizam do este sistema para aplicação de controle como Adaptive Sliding Mode Controller (Lu et al., 2018) e controle PID com sintonização automática utilizando lógica Fuzzy (Malayjerdi et al., 2018). Neste trabalho, o modelo do sistema será baseado em quatérnios e representado utilizando DAR. A partir dessa representação, serão utilizadas restrições na forma de LMIs compondo um problema de otimização convexo para o projeto sistemático do controlador. Este artigo contribui com a aplicação da DAR e subsequente projeto de controle utilizando LMIs para veículos com rodas omnidirecionais representados através de quatérnios. Os resultados serão ilustrados a partir de simulação numérica utilizando o software Matlab. Ainda, pode-se destacar que o método proposto é expansível para a futura consideração da saturação nos sinais de controle e incertezas paramétricas na dinâmica do sistema.

O artigo está estruturado da seguinte forma. Na Seção 2 será descrita a fundamentação teórica para a metodologia do trabalho, além da descrição do modelo do sistema utilizado. A seção 3 irá propor uma representação do sistema utilizando DAR. A Seção 4 descreverá a síntese do controlador, com base em LMIs. Na Seção 5 serão exibidos resultados obtidos a partir da simulações numéricas da aplicação do controle projetado. Por fim, na Seção 6 serão apresentadas as considerações finais sobre o artigo e propostas de trabalhos futuros.

Notação: $x_{i}$ representa o $i$-ésimo elemento de um vetor $\mathbf{x}$. $\dot{x}$ e $\ddot{x}$ significam a primeira e segunda derivadas temporais de $x$, respectivamente. A transposta de $A$ é representada por $A^{T} \cdot \operatorname{tr}(A)$ representa o traço da matriz A. He $\{A\}$ representa o bloco simétrico $A+A^{T}$. $\operatorname{diag}\{A, B\}$ representa o bloco da matriz diagonal formada por $A$ e $B$. Os elementos simétricos de uma matriz são representados por

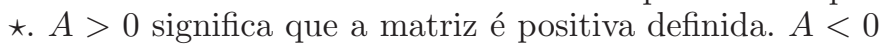
significa que a matriz é negativa definida. $I_{n}$ representa uma matriz identidade $n \times n$. $0_{n \times m}$ representa uma matriz de zeros $n \times m$. Para um conjunto politópico $\mathcal{X}$, o conjunto de todos os vértices de $\mathcal{X}$ é representado por $\mathcal{V}(\mathcal{X})$.

\section{PRELIMINARES}

Veículos omnidirecionais são veículos cujas rodas permitem o movimento em todas direções. Serão utilizadas rodas do tipo Mecanum, como as do conjunto visto na Figura 1. Um esquemático do veículo omnidirecional é apresentado na Figura 2. Nesta figura, podem ser observadas as coordenadas locais $\left(x_{l}, y_{l}, \psi_{l}\right)$ e globais $(x, y, \psi)$, além das características construtivas do sistema, onde $\alpha$ é o ângulo das rodas Mecanum, e $D$ e $W$ as distâncias do

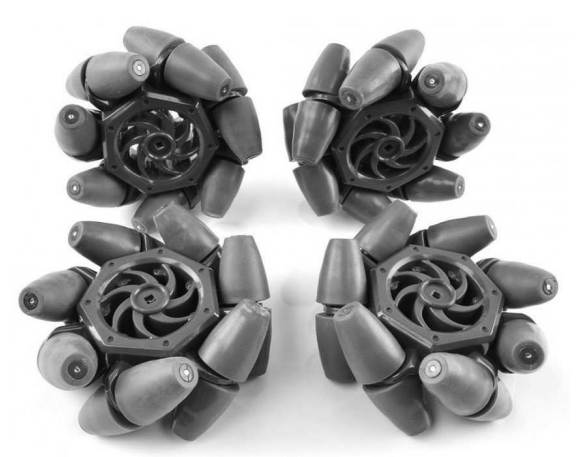

Figura 1. Conjunto de rodas Mecanum (Robotics, 2017). centro das rodas ao centro do veículo nas direções $y_{l}$ e $x_{l}$, respectivamente.

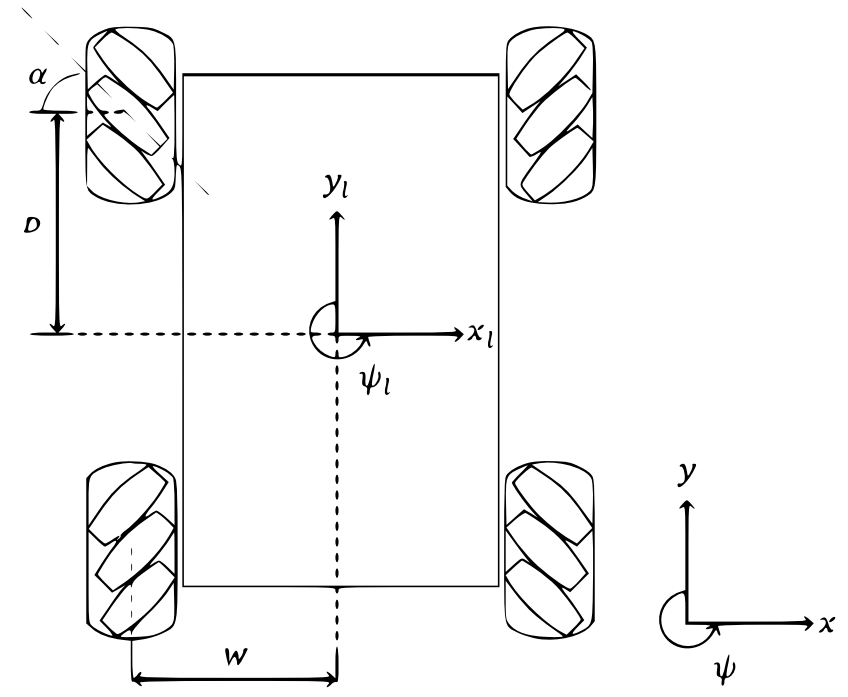

Figura 2. Esquemático do veículo omnidirecional. Adaptada de Won Yoon et al. (2015).

\subsection{Modelo de Coordenadas Locais}

O modelo em coordenadas locais do sistema pode ser descrito como (Lasevitch, 2014; Vancin, 2016):

$$
\left[\begin{array}{c}
\ddot{x}_{l} \\
\ddot{y}_{l} \\
\ddot{\psi}_{l}
\end{array}\right]=\left[\begin{array}{c}
c_{x} \dot{x}_{l} \\
c_{y} \dot{y}_{l} \\
c_{\psi} \dot{\psi}_{l}
\end{array}\right]+b\left[\begin{array}{cccc}
-c & c & -c & c \\
s & s & s & s \\
d & -d & -d & d
\end{array}\right]\left[\begin{array}{l}
u_{1} \\
u_{2} \\
u_{3} \\
u_{4}
\end{array}\right]
$$

onde $s:=\sin (\alpha), c:=\cos (\alpha)$ e $d:=\sqrt{D^{2}+W^{2}} ; c_{x}$ e $c_{y}$ são razões entre os respectivos coeficientes de atrito e a massa do veículo; $c_{\psi}$ é a razão entre coeficiente de atrito e momento de inércia do veículo; $\mathbf{u}=\left[\begin{array}{llll}u_{1} & u_{2} & u_{3} & u_{4}\end{array}\right]^{T}$ é o torque das rodas; e $b$ é uma constante que relaciona as entradas $\mathbf{u}$ à aceleração produzida pelos motores do veículo.

\subsection{Modelo de Coordenadas Globais baseado em Quatérnio}

Um quatérnio $\mathbf{q} \in \mathbb{R}^{4}$ pode ser representado pelo vetor:

$$
\mathbf{q}=\left[\begin{array}{llll}
\eta & \epsilon_{1} & \epsilon_{2} & \epsilon_{3}
\end{array}\right]^{T}=\left[\begin{array}{ll}
\eta & \boldsymbol{\epsilon}
\end{array}\right]^{T}
$$

Trata-se de um número hipercomplexo onde $\eta \in \mathbb{R}$ é a componente real do quatérnio e $\epsilon \in \mathbb{R}^{3}$ é a componente 
imaginária (Diebel, 2006). Um quatérnio unitário (que possui norma euclidiana 1), pode ser utilizado para representar a orientação de corpos rígidos, como será feito nesta aplicação. Representando o quatérnio em termos de rotação ângulo-eixo, temos:

$$
\mathbf{q}=\left[\begin{array}{c}
\cos \left(\frac{\psi}{2}\right) \\
\boldsymbol{r} \sin \left(\frac{\psi}{2}\right)
\end{array}\right]=\left[\begin{array}{l}
\eta \\
\boldsymbol{\epsilon}
\end{array}\right],\|\mathbf{q}\|=\sqrt{\eta^{2}+\boldsymbol{\epsilon}^{T} \boldsymbol{\epsilon}}=1,
$$

onde o vetor unitário $\boldsymbol{r} \in \mathbb{R}$ representa a direção em que a rotação $\psi$ ocorre (Salton et al., 2017).

Pode-se, também, definir uma matriz de rotação em termos de quatérnio, obtendo $R(\mathbf{q}) \in \mathbb{R}^{3 \times 3}$ como:

$$
R(\mathbf{q})=I_{3}+2 \eta S(\boldsymbol{\epsilon})+2 S^{2}(\boldsymbol{\epsilon}),
$$

onde $S(\cdot)$ representa a operação de produto vetorial na forma matricial:

$$
S(\mathbf{z})=\left[\begin{array}{ccc}
0 & -z_{3} & z_{2} \\
z_{3} & 0 & -z_{1} \\
-z_{2} & z_{1} & 0
\end{array}\right]
$$

Considerando que para um veículo omnidirecional que rotaciona apenas em torno do eixo vertical, temos que $\boldsymbol{r}=\left[\begin{array}{lll}0 & 0 & 1\end{array}\right]^{T}$. Desta forma, podemos reduzir o quatérnio para $\mathbf{q} \in \mathbb{R}^{2}$ :

$$
\mathbf{q}=\left[\begin{array}{l}
\cos \left(\frac{\psi}{2}\right) \\
\sin \left(\frac{\psi}{2}\right)
\end{array}\right]=\left[\begin{array}{l}
\eta \\
\epsilon
\end{array}\right]
$$

Além disso, obtém-se de (4) a matriz de rotação $R(\mathbf{q}) \in$ $\mathbb{R}^{2 \times 2}$ :

$$
R(\mathbf{q})=\left[\begin{array}{ll}
\cos (\psi) & -\sin (\psi) \\
\sin (\psi) & \cos (\psi)
\end{array}\right]=\left[\begin{array}{cc}
1-2 \epsilon^{2} & -2 \eta \epsilon \\
2 \eta \epsilon & 1-2 \epsilon^{2}
\end{array}\right]
$$

Com isso, o modelo em coordenadas globais do sistema é dado por:

$$
\left[\begin{array}{c}
\ddot{x} \\
\ddot{y} \\
\ddot{\psi}
\end{array}\right]=\left[\begin{array}{c}
c_{x} \dot{x} \\
c_{y} \dot{y} \\
c_{\psi} \dot{\psi}
\end{array}\right]+b\left[\begin{array}{cc}
R(\mathbf{q}) & 0 \\
0 & 1
\end{array}\right]\left[\begin{array}{cccc}
-c & c & -c & c \\
s & s & s & s \\
d & -d & -d & d
\end{array}\right]\left[\begin{array}{l}
u_{1} \\
u_{2} \\
u_{3} \\
u_{4}
\end{array}\right] .
$$

Também sabe-se que as derivadas de $\eta$ e $\epsilon$ são dadas por:

$$
\begin{aligned}
\dot{\eta} & =-\frac{1}{2} \epsilon \dot{\psi}, \\
\dot{\epsilon} & =\frac{1}{2} \eta \dot{\psi} .
\end{aligned}
$$

Tendo em vista que $\eta:=\cos (\psi / 2)$ e $\epsilon:=\sin (\psi / 2)$, conforme (6), é possível recuperar o ângulo de rotação $\psi$ através da identidade trigonométrica:

$$
\psi=2 \operatorname{arctg}\left(\frac{\epsilon}{\eta}\right) .
$$

Neste trabalho, considera-se sem perda de generalidade ${ }^{1}$ que o objetivo do controle é estabilizar a orientação e posição do veículo na origem do sistema de coordenadas.

\footnotetext{
1 Para orientar e posicionar arbitrariamente o veículo, basta considerar uma translação e rotação prévia do sistema de coordenadas para deslocar o orientação e posição desejada para a origem.
}

Assim, quando a rotação do sistema é representada através de quatérnio, para alcançar a orientação identidade $R(\mathbf{q})=I_{3}$ basta garantir a convergência da componente $\epsilon$ para zero. Desta forma a representação do sistema será simplificada considerando somente a dinâmica $\dot{\epsilon}$, onde

$$
\eta= \pm \sqrt{1-\epsilon^{T} \epsilon}
$$

será considerado como um parâmetro incerto dependente de $\epsilon$, conforme à restrição de norma apresentada em (3).

Para eliminar a dualidade no sinal deste parâmetro $\eta$, propõe-se utilizar a seguinte mudança de variável, tal como utilizado em Salton et al. (2017):

$$
\varepsilon=\operatorname{sign}(\eta) \epsilon,
$$

cuja derivada no tempo é dada por:

$$
\dot{\varepsilon}=\operatorname{sign}(\eta) \dot{\epsilon}+\delta_{D}(\eta) \dot{\eta} \epsilon
$$

Para evitar a descontinuidade do delta de Dirac $\delta_{D}(\eta)$, neste projeto o domínio de análise será limitado por $\eta \in$ $[-1,1]-\{0\}$. E neste domínio, $\dot{\varepsilon}$ pode ser reescrito como:

$$
\dot{\varepsilon}=\frac{|\eta|}{2} \dot{\psi}, \quad|\eta| \in(0,1]
$$

Além disso, é possível reescrever $R(\mathbf{q})$, de forma equivalente, em termos de $|\eta|$ e $\varepsilon$ :

$$
\begin{gathered}
R(|\eta|, \varepsilon)=\left[\begin{array}{cc}
1-2(\operatorname{sign}(\eta) \epsilon)^{2} & -2|\eta| \operatorname{sign}(\eta) \epsilon \\
2|\eta| \operatorname{sign}(\eta) \epsilon & 1-2(\operatorname{sign}(\eta) \epsilon)^{2}
\end{array}\right] \\
=\left[\begin{array}{cc}
1-2 \varepsilon^{2} & -2|\eta| \varepsilon \\
2|\eta| \varepsilon & 1-2 \varepsilon^{2}
\end{array}\right]
\end{gathered}
$$

Assim, o sistema descrito em (8) pode ser reescrito da seguinte forma:

$$
\left[\begin{array}{c}
\ddot{x} \\
\ddot{y} \\
\ddot{\psi}
\end{array}\right]=\left[\begin{array}{c}
c_{x} \dot{x}+b( \\
c_{y} \dot{y}+b( \\
(-c \beta-s \gamma) u_{1}+(c \beta-s \gamma) u_{2} \\
c_{\psi} \dot{\psi}+b\left(d u_{1}-d u_{2}\right. \\
\left.+(-c \beta-s \gamma) u_{3}+(c \beta-s \gamma) u_{4}\right) \\
\left.+(-c \gamma+s \beta) u_{3}+(c \gamma+s \beta) u_{4}\right) \\
\left.-d u_{3}+d u_{4}\right)
\end{array}\right] .
$$

onde $\beta:=1-2 \varepsilon^{2}$ e $\gamma:=2|\eta| \varepsilon$.

\section{REPRESENTAÇÃO ALGÉBRICO-DIFERENCIAL}

A DAR pode ser considerada conforme descrita em Trofino (2000):

$$
\left\{\begin{array}{l}
\dot{\mathbf{x}}=A_{1}(\mathbf{x}, \boldsymbol{\delta}) \mathbf{x}+A_{2}(\mathbf{x}, \boldsymbol{\delta}) \boldsymbol{\xi}(\mathbf{x}, \boldsymbol{\delta}, \mathbf{u})+B(\mathbf{x}, \boldsymbol{\delta}) \mathbf{u} \\
0=\Omega_{1}(\mathbf{x}, \boldsymbol{\delta}) \mathbf{x}+\Omega_{2}(\mathbf{x}, \boldsymbol{\delta}) \boldsymbol{\xi}(\mathbf{x}, \boldsymbol{\delta}, \mathbf{u})+\Omega_{3}(\mathbf{x}, \boldsymbol{\delta}) \mathbf{u}
\end{array}\right.
$$

onde $\mathbf{x} \in \mathbb{R}^{n}$ é o vetor de estados do sistema; $\boldsymbol{\xi}(\mathbf{x}, \boldsymbol{\delta}, \mathbf{u}) \in$ $\mathbb{R}^{m}$ é um mapa vetorial arbitrário de termos não lineares; $\mathbf{u} \in \mathbb{R}^{p}$ representa as entradas do sistema; $A_{1}(\mathbf{x}, \boldsymbol{\delta}) \in$ $\mathbb{R}^{n \times n}, A_{2}(\mathbf{x}, \boldsymbol{\delta}) \in \mathbb{R}^{n \times m}$ e $B(\mathbf{x}, \boldsymbol{\delta}) \in \mathbb{R}^{n \times p}$ são matrizes afim em $(\mathbf{x}, \boldsymbol{\delta})$ associadas à estrutura do sistema; e $\Omega_{1}(\mathbf{x}, \boldsymbol{\delta}) \in \mathbb{R}^{m \times n}, \Omega_{2}(\mathbf{x}, \boldsymbol{\delta}) \in \mathbb{R}^{m \times m}$ e $\Omega_{3}(\mathbf{x}, \boldsymbol{\delta}) \in \mathbb{R}^{m \times p}$ são matrizes afim em de $(\mathbf{x}, \boldsymbol{\delta})$ usadas para expressar relações adicionais dos vetores $\mathbf{x}$ e $\boldsymbol{\xi}(\mathbf{x}, \boldsymbol{\delta}, \mathbf{u})$. Para recuperação do sistema original a partir da representação em DAR, é possível utilizar a seguinte relação ${ }^{2}$ :

$$
\dot{\mathbf{x}}=\left(A_{1}-A_{2} \Omega_{2}^{-1} \Omega_{1}\right) \mathbf{x}+\left(B-A_{2} \Omega_{2}^{-1} \Omega_{3}\right) \mathbf{u} .
$$

2 Para simplificar a notação, as matrizes e vetores passarão a ser representados sem suas dependências de $\mathbf{x}$ e $\boldsymbol{\delta}$ 
Então, para representação do sistema (16) na forma (17), definem-se os vetores $\mathbf{x}$ e $\boldsymbol{\xi}$ como:

$$
\begin{gathered}
\mathbf{x}=\left[\begin{array}{llllll}
x & \dot{x} & y & \dot{y} & \varepsilon & \dot{\psi}
\end{array}\right]^{T}=\left[\begin{array}{llllll}
x_{1} & x_{2} & x_{3} & x_{4} & x_{5} & x_{6}
\end{array}\right]^{T} \mathrm{e} \\
\boldsymbol{\xi}=\left[\begin{array}{lllllll}
x_{5} & u_{1} & x_{5} & u_{2} & x_{5} & u_{3} & x_{5} u_{4}
\end{array}\right]^{T} .
\end{gathered}
$$

Essa escolha de estados permite representar $|\eta(t)|$ como uma função de $\mathbf{x}$. Além disso, a componente $|\eta(t)|$ será tratada como um parâmetro incerto $\delta(\mathbf{x})=\sqrt{1-x_{5}^{T} x_{5}}$, conforme a restrição da norma unitária do quatérnio (3).

Finalmente, as seguintes matrizes são utilizadas para expressar o sistema na forma (17):

$$
\begin{aligned}
A_{1}= & {\left[\begin{array}{llllll}
0 & 1 & 0 & 0 & 0 & 0 \\
0 & c_{x} & 0 & 0 & 0 & 0 \\
0 & 0 & 0 & 1 & 0 & 0 \\
0 & 0 & 0 & c_{y} & 0 & 0 \\
0 & 0 & 0 & 0 & 0 & \frac{\delta}{2} \\
0 & 0 & 0 & 0 & 0 & c_{\psi}
\end{array}\right], B=\left[\begin{array}{cccc}
0 & 0 & 0 & 0 \\
-b c & b c & -b c & b c \\
0 & 0 & 0 & 0 \\
b s & b s & b s & b s \\
0 & 0 & 0 & 0 \\
b d & -b d & -b d & b d
\end{array}\right], \quad(20) } \\
A_{2}=2 b & {\left[\begin{array}{cccc}
0 & 0 & 0 \\
\left(c x_{5}-s \delta\right) & -\left(c x_{5}+s \delta\right) & \left(c x_{5}-s \delta\right) & -\left(c x_{5}+s \delta\right) \\
0 & 0 & 0 & 0 \\
-\left(c \delta+s x_{5}\right) & \left(c \delta-s x_{5}\right) & -\left(c \delta+s x_{5}\right) & \left(c \delta-s x_{5}\right) \\
0 & 0 & 0 & 0 \\
0 & 0 & 0 & 0
\end{array}\right], } \\
& \Omega_{1}=0_{4 \times 6}, \quad \Omega_{2}=I_{4}, \quad \Omega_{3}=-x_{5} I_{4} .
\end{aligned}
$$

A variável de estado $x_{5}$, relativa ao quatérnio, será aqui tratada no domínio de interesse $x_{5} \in[-\sin (\bar{\psi} / 2), \sin (\bar{\psi} / 2)]$, onde $0 \leq \bar{\psi}<\pi$ representa o ângulo máximo admissível do veículo em relação à origem. $\mathrm{O}$ conjunto de validade de todos os estados da DAR é portanto definido como:

$$
\mathcal{X}=\left\{\mathbf{x} \in \mathbb{R}^{6}:|\boldsymbol{\rho} \mathbf{x}| \leq 1\right\}, \boldsymbol{\rho}=\left[\begin{array}{llllll}
0 & 0 & 0 & 0 & \bar{x}_{5}^{-1} & 0
\end{array}\right] .
$$

Devido à restrição (3), nota-se que $\mathbf{x} \in \mathcal{X}$ implica em $\delta(\mathbf{x}) \in \mathcal{D}=[\cos (\bar{\psi} / 2), 1]$.

\section{PROJETO SISTEMÁTICO DO CONTROLADOR}

Visando o projeto sistemático do controle do sistema com garantias formais de estabilidade, o seguinte lema extraído de Khalil (2002) será utilizado.

Lema 1. Considere um sistema $\dot{\mathbf{x}}=f(\mathbf{x})$ onde $f: \mathcal{X} \mapsto$ $\mathbb{R}^{n}$ com ponto de equilíbrio $\mathbf{x}=0$. Suponha que exista uma função $V: \mathcal{X} \mapsto \mathbb{R}$ tal que:

$$
\begin{gathered}
V(0)=0, V(\mathbf{x})>0 \forall \mathbf{x} \in \mathcal{X}, \mathbf{x} \neq 0, \\
\dot{V}(\mathbf{x})<0 \forall \mathbf{x} \in \mathcal{X}, \mathbf{x} \neq 0 \\
\mathcal{R}:=\left\{\mathbf{x} \in \mathbb{R}^{n}: V(\mathbf{x}) \leq 1\right\} \subset \mathcal{X} .
\end{gathered}
$$

Então, as trajetórias $\mathbf{x}(t)$ tendem assintoticamente à origem $\forall \mathbf{x}(0) \in \mathcal{R}$.

É proposto aqui o uso de um controlador por realimentação de estados, na forma

$$
\mathbf{u}=K \mathbf{x},
$$

para $K \in \mathbb{R}^{4 \times 6}$. Considera-se, também, que todos os estados são medidos e estão acessíveis. Para projetar a matriz de ganhos $K$, leva-se adicionalmente em consideração os seguintes critérios de desempenho, além da estabilidade assintótica: (a) As trajetórias $\mathbf{x}(t)$ do sistema convergem exponencialmente para a origem com taxa de decaimento mais rápida que $\lambda>0$, ou seja, $\exists \kappa:\|\mathbf{x}(t)\| \leq \kappa \mathrm{e}^{-\lambda t}$ $\forall \mathbf{x}(0) \in \mathcal{R}$;

(b) Os autovalores da matriz $\left(A_{1}+B K\right)$ estão contidos no disco $\{s \in \mathbb{C}:|s|<r\} \forall(\mathbf{x}, \delta) \in \mathcal{X} \times \mathcal{D}$ para um raio $r>0$.

Por meio do parâmetro $\lambda$ descrito no critério (a), é possível especificar a rapidez com que o controle deve estabilizar a posição e orientação do veículo. Por outro lado, o parâmetro $r$, introduzido em (b), permite limitar a magnitude dos ganhos de realimentação, evitando produção de um controle demasiadamente agressivo.

Com base nos critérios estabelecidos, é proposto o seguinte teorema para síntese de controle, o qual é adaptado de Salton et al. (2017) e Castro (2019).

Teorema 1. Considere o sistema em (16) e sua representação em DAR (17) sujeito à lei de controle (27). Suponha que exista uma matriz $\widehat{P}=\widehat{P}^{T} \in \mathbb{R}^{6 \times 6}$ e uma matriz livre $\widehat{L} \in \mathbb{R}^{4 \times 4}$ que satisfaçam as seguintes inequações para todo $(\mathbf{x}, \boldsymbol{\delta})$ avaliados nos vértices $\mathcal{V}(\mathcal{X}) \times \mathcal{V}(\mathcal{D})$ :

$$
\begin{gathered}
\widehat{P}>0 \\
\operatorname{He}\left\{\left[\begin{array}{cc}
A_{1} \widehat{P}+B \widehat{K}+\lambda \widehat{P} & A_{2} \widehat{L} \\
\Omega_{1} \widehat{P}+\Omega_{3} \widehat{K} & \Omega_{2} \widehat{L}
\end{array}\right]\right\}<0 \\
{\left[\begin{array}{cc}
1 & \rho \widehat{P} \\
\star & \widehat{P}
\end{array}\right]>0} \\
{\left[\begin{array}{cc}
-r \widehat{P} & A_{1} \widehat{P}+B \widehat{K} \\
\star & -r \widehat{P}
\end{array}\right]<0}
\end{gathered}
$$

Então, as trajetórias do sistema em malha fechada com $K=\widehat{K} \widehat{P}^{-1}$ convergem assintoticamente para a origem $e$ satisfazem os critérios (a) e (b) para toda condição inicial $\mathbf{x}(0)$ que inicie em:

$$
\mathcal{R}=\left\{\mathbf{x} \in \mathbb{R}^{6}: \mathbf{x}^{T} \widehat{P}^{-1} \mathbf{x} \leq 1\right\} .
$$

Prova. Considere uma função candidata de Lyapunov conforme:

$$
V(\mathbf{x})=\mathbf{x}^{T} P \mathbf{x}
$$

onde $P>0$ para que (24) seja verdade. Ao derivar (33), ao longo das trajetórias do sistema (16) representado em DAR, obtém-se:

$$
\dot{V}(\mathbf{x}, \boldsymbol{\delta})=\left[\mathbf{x}^{T} \boldsymbol{\xi}^{T}\right] \operatorname{He}\left\{\left[\begin{array}{cc}
P\left(A_{1}+B K\right) & P A_{2} \\
0 & 0
\end{array}\right]\right\}\left[\begin{array}{l}
\mathbf{x} \\
\boldsymbol{\xi}
\end{array}\right] .
$$

Agora suponha que a seguinte inequação seja satisfeita para todo $(\mathbf{x}, \delta) \in \mathcal{X} \times \mathcal{D}$ :

$$
\dot{V}(\mathbf{x}, \boldsymbol{\delta})+2 \lambda V(\mathbf{x})+\operatorname{He}\left\{\boldsymbol{\xi}^{T} L\left[\Omega_{1}+\Omega_{3} K \Omega_{2}\right]\left[\begin{array}{l}
\mathbf{x} \\
\boldsymbol{\xi}
\end{array}\right]\right\}<0,
$$

ou equivalentemente:

$$
\mathrm{He}\left\{\left[\begin{array}{cc}
P\left(A_{1}+B K\right)+\lambda P & P A_{2} \\
L\left(\Omega_{1}+\Omega_{3} K\right) & L \Omega_{2}
\end{array}\right]\right\}<0 .
$$

Dado que $\lambda \geq 0, V(\mathbf{x})>0$ e $\left(\Omega_{1}+\Omega_{3} K\right) \mathbf{x}+\Omega_{2} \boldsymbol{\xi}=0$, segue que (35) implica (25). Realizando a pré- e pósmultiplicação de (36) por $\operatorname{diag}\left\{P^{-1}, L^{-T}\right\}$, obtém-se a relação (29) ao considerar as trocas de variáveis $\widehat{P}=P^{-1}$ 


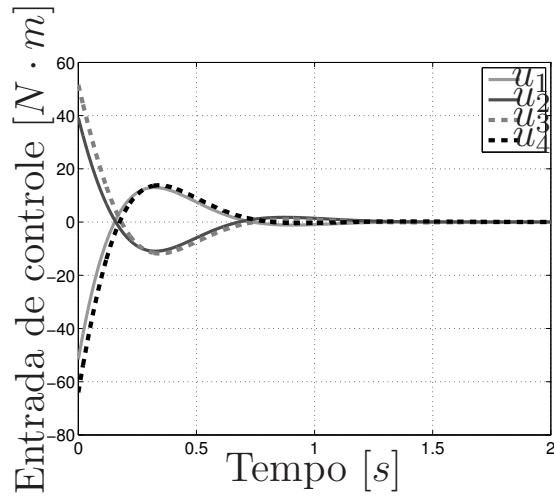

(a) Sinal de entrada de controle.

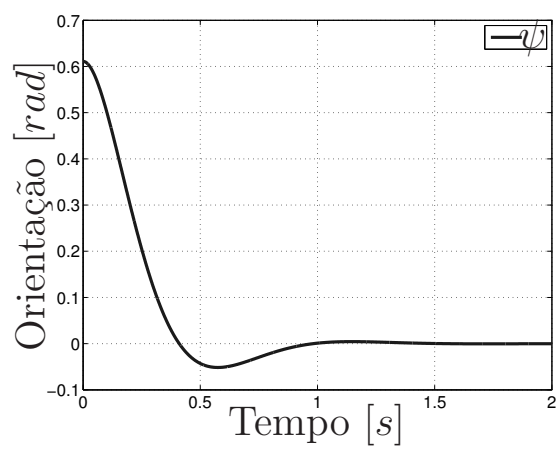

(d) Trajetória da orientação $\psi$.

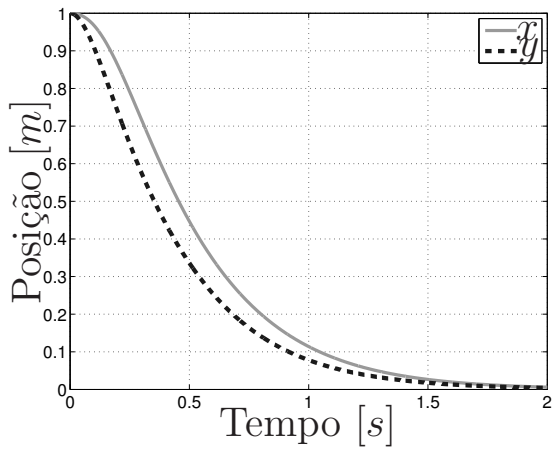

(b) Trajetória das posições $x$ e $y$.

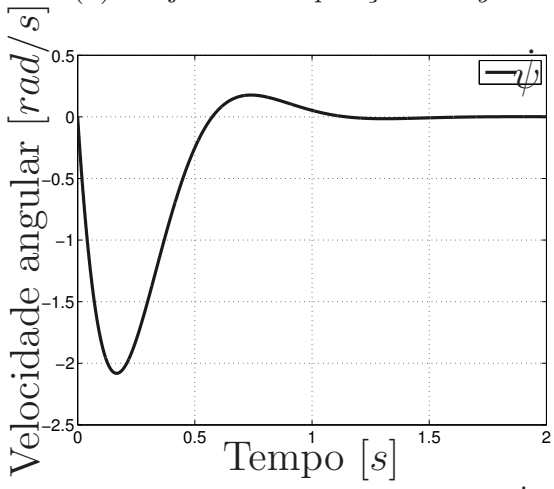

(e) Trajetória da velocidade angular $\dot{\psi}$.

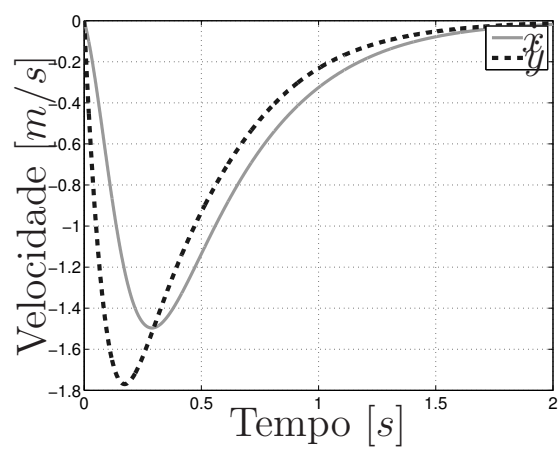

(c) Trajetória das velocidades $\dot{x}$ e $\dot{y}$.

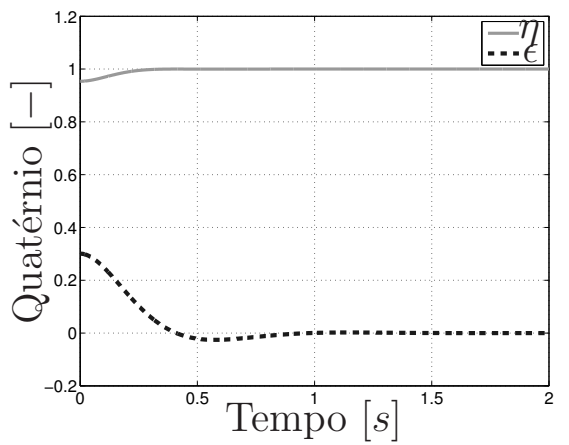

(f) Trajetórias do quatérnio $\eta$ e $\epsilon$.

Figura 3. Trajetórias obtidas a partir da simulação

e $\widehat{L}=L^{-T}$. Da mesma forma, ao realizar a pré- e pósmultiplicação de $P>0$ por $P^{-1}$, verifica-se a relação (28).

Conforme requirido pelo Lema 1 , para que $\mathcal{R} \subset \mathcal{X}$ é necessário garantir adicionalmente que:

$$
\mathbf{x}^{T} \boldsymbol{\rho}^{T} \boldsymbol{\rho} \mathbf{x}<\mathbf{x}^{T} P \mathbf{x} \leq 1 \Leftrightarrow P-\boldsymbol{\rho}^{T} \boldsymbol{\rho}>0 .
$$

Aplicando o complemento de Schur, esta última relação pode ser expressa como

$$
\left[\begin{array}{ll}
1 & \rho \\
\star & P
\end{array}\right]>0
$$

Pré- e pós-multiplicando (38) por $\operatorname{diag}\left\{1, P^{-1}\right\}$, obtém-se (30). Logo, o Lema 1 é satisfeito pelas condições (28), (29) e (30), o que garante que todas trajetórias iniciadas em $\mathcal{R}$ irão convergir assintoticamente para a origem.

Da desigualdade apresentada em (35), segue complementarmente que

o que implica:

$$
\dot{V}(\mathbf{x}, \boldsymbol{\delta})<-2 \lambda V(\mathbf{x})
$$

$$
V(\mathbf{x}(t)) \leq V(\mathbf{x}(0)) \mathrm{e}^{-2 \lambda t} \leq \mathrm{e}^{-2 \lambda t} \forall \mathbf{x}(0) \in \mathcal{R} .
$$

Assim sendo, esta condição garante o decaimento exponencial das trajetórias iniciadas em $\mathcal{R}$, conforme o critério (a), visto que (Castro, 2019):

$$
\sqrt{P_{\min }}\|\mathbf{x}(t)\| \leq \sqrt{V(\mathbf{x}(t))} \leq \mathrm{e}^{-\lambda t},
$$

onde $P_{\min }$ é o menor autovalor da matriz $P$.

Por fim, o critério (b) é considerado pela adição da seguinte condição (Boyd et al., 1994):

$$
\left[\begin{array}{cc}
-r P & P\left(A_{1}+B K\right) \\
\star & -r P
\end{array}\right]<0
$$

onde pré- e pós-multiplicando por $\operatorname{diag}\left\{P^{-1}, P^{-1}\right\}$ obtémse a relação $(31)$.
Por argumentos de convexidade, sabe-se que as relações (29) e (31) são válidas para todo $\mathcal{X} \times \mathcal{D}$ se satisfeitas apenas nos vértices $\mathcal{V}(\mathcal{X}) \times \mathcal{V}(\mathcal{D})$.

A partir da definição da região de atração em (32), concluise que a maximização do traço de $\widehat{P}$ implica também na maximização dos eixos do conjunto elipsoidal $\mathcal{R}$. Sendo assim, o cálculo do ganho $K$ que maximiza a região $\mathcal{R}$ pode ser resolvido pelo seguinte problema de otimização convexo baseado no Teorema 1:

$$
\begin{array}{ll}
\underset{\widehat{P}, \widehat{L}, \widehat{K}}{\operatorname{minimizar}} & \operatorname{tr}(N) \\
\text { sujeito a } & (28),(29),(30),(31),\left[\begin{array}{ll}
N & I_{6} \\
I_{6} & \widehat{P}
\end{array}\right]>0 .
\end{array}
$$

Aplicando o complemento de Schur na desigualdade matricial introduzida em (43), onde $N \in \mathbb{R}^{6 \times 6}$ é uma matriz simétrica e $I_{6} \in \mathbb{R}^{6 \times 6}$ é uma matriz identidade, resulta-se que $N>\widehat{P}^{-1}$. Logo, lembrando que $\widehat{P}^{-1}=P$, minimizar o traço de $N$ implica em maximizar a região de atração $\mathcal{R}$, pois $\operatorname{tr}(N)>\operatorname{tr}(P)$.

\section{EXEMPLO NUMÉRICO}

A simulação foi realizada no software MATLAB utilizando o modelo apresentado em (16) e o controle descrito na seção 4. A Tabela 1 mostra os parâmetros utilizados na simulação, que incluem as condições iniciais e as constantes das características construtivas do modelo. As condições iniciais foram escolhidas de modo que $\mathbf{x}(0)^{T} P \mathbf{x}(0)=$ $0.9880 \leq 1$, ou seja, próximas do limite da região de atração em que foi provada a funcionalidade do controle.

A Figura 2 contém as trajetórias obtidas a partir da simulação: Figura 2.a traz o sinal das entradas do sistema; 
Tabela 1. Parâmetros da simulação

\begin{tabular}{l|c|l|c}
\hline Parâmetro & Valor & Parâmetro & Valor \\
\hline$c_{x}, c_{y}, c_{\psi}$ & $-0.1 s^{-1}$ & $b$ & $1(\mathrm{~kg} \cdot \mathrm{m})^{-1}$ \\
$\alpha$ & $45^{\circ}$ & $D, W$ & $0.1 \mathrm{~m}$ \\
$\bar{\psi}$ & $45^{\circ}$ & $r$ & 8 \\
$\lambda$ & $2 s^{-1}$ & $x(0), y(0)$ & $1 \mathrm{~m}$ \\
$\psi(0)$ & $35^{\circ}$ & $\dot{x}(0), \dot{y}(0), \dot{\psi}(0)$ & 0 \\
$\eta(0)$ & $\sin (\psi(0) / 2)$ & $\epsilon(0)$ & $\cos (\psi(0) / 2)$ \\
\hline
\end{tabular}

Figura 2.b mostra a trajetória das posições $x$ e $y$ tendendo para origem, como previsto no Teorema 1 que satisfaz o Lema 1; o mesmo é observado em relação às velocidades, na figura 2.c; as trajetórias da orientação e velocidade angular são vistas nas Figuras 2.d e 2.e, respectivamente; a Figura 2.f mostra a trajetória de $\eta$ tendendo a 1 e $\epsilon$ tendendo a 0 , conforme previsto em (11); por fim, a Figura 4 mostra a animação da simulação com frames sobrepostos a cada 0.2 segundos, de 0 a 2 segundos, mostrando a trajetória espacial do veículo nesse intervalo de tempo.

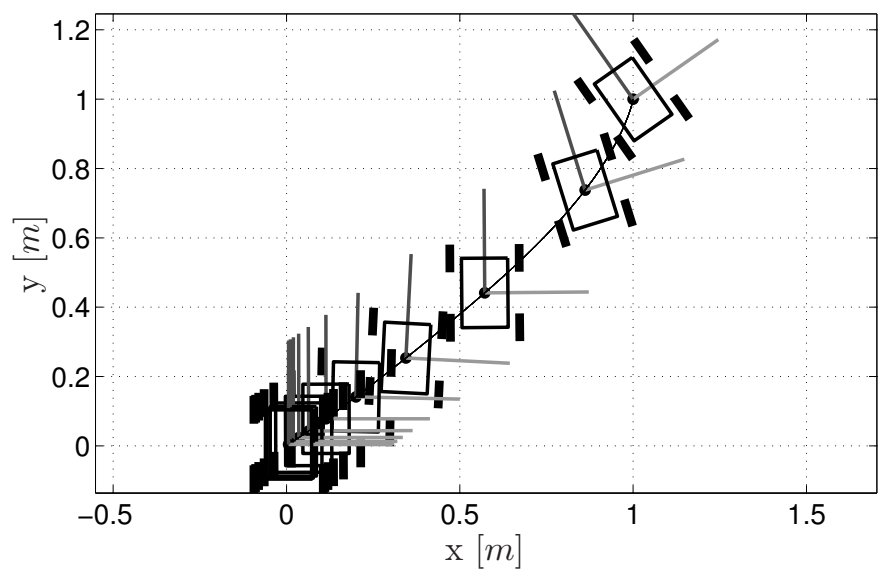

Figura 4. Trajetória espacial do veículo. Animação com frames sobrepostos a cada 0.2 segundos.

\section{CONCLUSÃO E TRABALHOS FUTUROS}

O presente trabalho apresentou um método de controle de realimentação de estados aplicado a um sistema real não linear: um veículo omnidirecional com rodas mecanum. O modelo do sistema foi descrito utilizando quatérnios, o que tornou as equações do sistema racionais, permitindo sua representação na forma de DAR. Por sua vez, esta representação tornou possível a realização da síntese do controlador no framework de LMIs.

Para trabalhos futuros pretende-se adicionar ao modelo saturação nos atuadores e incertezas paramétricas, que pode ser realizado de forma relativamente fácil dada a característica de extensibilidade do método proposto. Também é sugerida a análise de desempenho da aplicação do método proposto na presença de ruído de medição e dinâmica nãomodelada. Além disso, propõe-se a realimentação das nãlinearidades na DAR na lei de controle.

\section{REFERÊNCIAS}

Boyd, S., Ghaoui, L., Feron, E., and Balakrishnan, V. (1994). Linear matrix inequalities in system and control theory. SIAM studies in applied mathematics. Society for Industrial and Applied Mathematics.
Castro, R.S. (2019). Output regulation of rational nonlinear systems with input saturation. Ph.D. thesis, Escola de Engenharia. Programa de Pós-Graduação em Engenharia Elétrica.

Castro, R.S., Flores, J.V., and Salton, A.T. (2017). Stability analysis of output regulated rational nonlinear systems. IFAC-PapersOnLine, 50(1), 8214-8219.

Coutinho, D.F., Bazanella, A.S., Trofino, A., and e Silva, A.S. (2004). Stability analysis and control of a class of differential-algebraic nonlinear systems. International Journal of Robust and Nonlinear Control, 14(16), 13011326.

Coutinho, D.F., de Souza, C.E., and Trofino, A. (2009). Stability analysis of implicit polynomial systems. IEEE Transactions on Automatic Control, 54(5), 1012-1018.

Coutinho, D., Fu, M., Trofino, A., and Danès, P. (2008). $\mathcal{L}_{2}$-gain analysis and control of uncertain nonlinear systems with bounded disturbance inputs. International Journal of Robust and Nonlinear Control, 18(1), 88-110.

Diebel, J. (2006). Representing attitude: Euler angles, unit quaternions, and rotation vectors.

Khalil, H. (2002). Nonlinear Systems. Pearson Education. Prentice Hall.

Lasevitch, H.R.R. (2014). Controle não-linear do veículo omni-direcional via servovisão e sensor inercial. Master's thesis, Programa de Pós-Graduação em Engenharia Elétrica. Faculdade de Engenharia.

Lu, X., Zhang, X., Zhang, G., and Jia, S. (2018). Design of adaptive sliding mode controller for four-mecanum wheel mobile robot. In 2018 37th Chinese Control Conference (CCC), 3983-3987.

Malayjerdi, E., Kalani, H., and Malayjerdi, M. (2018). Selftuning fuzzy pid control of a four-mecanum wheel omnidirectional mobile platform. In Iranian Conference on Electrical Engineering (ICEE), 816-820.

Naing, M.Y., Oo, A.S., Nilkhamhang, I., and Than, T. (2019). Development of computer vision-based movement controlling in mecanum wheel robotic car. In 2019 First International Symposium on Instrumentation, Control, Artificial Intelligence, and Robotics (ICASYMP), 45-48.

Oliveira, M.Z., da Silva, J.M.G., and Coutinho, D. (2012). State feedback design for rational nonlinear control systems with saturating inputs. In 2012 American Control Conference (ACC), 2331-2336.

Robotics, V. (2017). Vex 4" mecanum wheel set (2x left, $2 \mathrm{x}$ right). http://www.robotshop.com/en/ mecanum-wheel-set.html. Acessado em: 02/11/2017.

Salton, A.T., Castro, R.S., Borges, B.S., Flores, J.V., and Coutinho, D.F. (2017). Semidefinite programming solution to the spacecraft analysis and control problem. IFAC-PapersOnLine, 50(1), 3959 - 3964.

Trofino, A. (2000). Robust stability and domain of attraction of uncertain nonlinear systems. In Proceedings of the 2000 American Control Conference. ACC (IEEE Cat. No.00CH36334), volume 5, 3707-3711 vol.5.

Vancin, P.H. (2016). Fusão de imagens e sensores inerciais para a estimação e controle de veículos autônomos. Master's thesis, Programa de Pós-Graduação em Engenharia Elétrica. Faculdade de Engenharia.

Won Yoon, S., Park, S.B., and Kim, J. (2015). Kalman filter sensor fusion for mecanum wheeled automated guided vehicle localization. Journal of Sensors, 2015. 